\title{
Wrinkling and head shape as coordinated sources of age-level information
}

\author{
LEONARD S. MARK \\ University of Connecticut, Storrs, Connecticut 06268 \\ JOHN B. PITTENGER and HELEN HINES \\ University of Arkansas, Little Rock, Arkansas 72204 \\ and

\begin{abstract}
CLAUDIA CARELLO, ROBERT E. SHAW, and JAMES T. TODD
University of Connecticut, Storrs, Connecticut 06268
\end{abstract}

\begin{abstract}
Changes in the shape of a human head and the development of facial wrinkles were examined as potential sources of information about age level. In Experiment 1, subjects estimated the ages of faces that had been produced by systematically manipulating characteristic head shapes and levels of wrinkles associated with ages $15,30,50$, and 70 years. The results indicated that observers used both sources of craniofacial change in making age estimates; but the effect of either source of change on perceived age depended upon the level of the other source of change. In Experiment 2, subjects' ratings of the apparent conflict between levels of head shape and wrinkles further substantiated the conclusion that observers are sensitive to the coordination of products of the two sources of change. These findings suggest that the information specifying perceived age level is a complex relationship among different types of craniofacial change.
\end{abstract}

As people become older, their faces are subject to many changes: Throughout roughly the first 20 years of life, the head undergoes a global remodeling that effects a marked increase in the size of the facial mask (face) relative to the cranium (Enlow, 1968; Hogarth, 1965; Moore \& Lavelle, 1974), in adulthood, wrinkles, creases, bags, and wattles develop in response to biomechanical stresses (such as gravity, characteristic facial expressions, and facial postures) and chemical changes in the structure of collagen fibers (Kohn, 1971; Viidik, 1973); progressive changes in skin texture and hair color, as well as loss of teeth (edentation) and hair (balding) also occur frequently with advancing years. Each of these characteristics can be said to provide perceptual information about a person's age precisely because each is produced by a continuous change resulting from physical and biomechanical stresses occurring throughout the lifespan. Moreover, these changes do not take place independently of each other (Enlow,

This research was supported by grants from the National Institute of Dental Research (DE-1RO-DE04990-01), the Donaghey Foundation, and the University of Connecticut Research Foundation. The authors wish to thank Carl Cross and Jennipher Boone for assistance in collecting the data and Watson-Guptill Publishers for permission to use profiles from Burne Hogarth's Drawing the Human Face. Leonard S. Mark and John B. Pittenger should be recognized as senior authors. Requests for reprints should be sent to L. S. Mark, Psychology Department, U-20, University of Connecticut, Storrs, Connecticut 06268.
1968; Pick, 1949; cf. Hogarth, 1965); they are, within broad limits, temporally coordinated, since they are produced by biological processes that are themselves temporally coordinated, often as a result of a common force or stress acting upon tissue (Enlow, 1968; Pick, 1949). Thus, change in a given source of agelevel information is typically accompanied by changes in other age-level characteristics. Rarely do growth or aging characteristics reflect vastly different age levels unless facial appearance has been marred by an accident, a growth-related anomaly, or intentionally altered by cosmetic treatment or surgery. Moreover, when the undisturbed course of growth or aging does result in a marked discoordination of two or more aging characteristics, it is usually an indication of an abnormality. In some instances, such as progeria, a type of dwarfism which can produce wrinkling in a young child's skin, the abnormality is perceived as such (cf. Pick, 1949).

To date, our research on the perception of craniofacial growth and aging has focused on a single agerelated change- the global remodeling (shape transformation) of the craniofacial complex due to growth. The primary aim of this work has been to identify a mathematical transformation that produces the appearance of craniofacial growth when applied to any human head. Pittenger and Shaw (1975) examined two prospective "growth transformations," cardioidal strain $\left[\theta^{\prime}=\theta, \mathrm{r}^{\prime}=\mathrm{r}(1-\mathrm{k} \sin \theta)\right.$, where $\mathrm{k}$ is a free parameter] and affine shear $\left(y^{\prime}=y, x^{\prime}=\right.$ 
$\mathrm{x}+\mathrm{y} \tan \theta$, where $\theta$ is a free parameter). Different amounts of each transformation were applied to the profile of a 10-year-old boy, and the effects of the two transformations on perceived age level were compared. The outcome of an age judgment task (Experiment 1) showed that the cardioidal strain transformation had a marked effect on the perceived age level of the profile, in contrast to affine shear, which had a relatively weak effect. A second study (Mark, 1979) compared the perceptual consequences of cardioidal strain and other prospective "growth transformations" to actual growth, using a variety of converging tasks. The results of these experiments suggest that the perceptual effects produced by cardioidal strain are nearly equivalent to those produced by actual growth.

These findings have been buttressed and clarified by the outcomes of two related biological studies. In the first, the cardioidal strain transformation was quite successful in predicting the morphological outcome of actual growth. Profile tracings of lateral headplates ( $x$-rays) of young children could be transformed using cardioidal strain such that the transformed profile for each child closely matched the profiles of that child resulting from actual growth (Todd, Mark, Shaw, \& Pittenger, in press). A second line of research (Todd et al., in press) attempted to delineate specific dynamic forces that are responsible for the morphological change modeled by cardioidal strain. This analysis suggested that the change in craniofacial shape described by the cardioidal strain transformation is a consequence of the pressure gradient inside the human head due to gravity. To summarize, perceptual and biological evidence indicates that the cardioidal strain transformation is "perceptually equivalent" to the effects of the gravitational pressure distribution on the growing head (Mark, 1979).

By itself, however, this work does not address issues related to the perception of the age level of a face. Specifically, how do people evaluate the overall product of various growth and aging processes in making judgments about a person's age?' Since many types of craniofacial changes are potentially informative about the age of an individual, at least two questions should be raised: (1) What information is used in the perception of age level? (2) Is one source of information more important than others in making age judgments? Experiment 1 examined the relative importance of two general sources of agerelated changes: (1) head shape, which includes changes in facial proportions and profile contour, and (2) wrinkling, which refers to all changes in skin appearance (wrinkles, wattles, bags, creases, skin texture). ${ }^{2}$ As an initial hypothesis, we might expect that both types of change would vary in the amount of information conveyed throughout the life span:
Under normal circumstances, wrinkling does not begin prior to the 30th year and, therefore, should be relatively uninformative compared to changes in head shape during that period. But after the age of 30 , the relative importance of head shape and wrinkling should be reversed; most of the changes in the facial profile have been completed by age 30 (with the exception of those resulting from edentation), while the formation of wrinkles, wattles, bags, creases, etc., has just started. The first experiment tested this hypothesis by asking people to estimate the age of faces that were constructed by systematically varying the ages portrayed by head shape and wrinkling.

Another potentially useful source of age-level information exists in the relations among the products of individual age-related changes. That is, various changes are coordinate to one another. We asked, therefore, if observers would be more confident in their age judgments of a given face if the head shape and the wrinkles portrayed roughly the same age level than if they portrayed very different ages. Experiment 2 investigated observers' sensitivity to conflicts between "head-shape age" and "wrinkle age." If head shape and wrinkles provide salient age-level information, it is reasonable to suppose that observers would notice conflicts in the ages specified by the two sources of change.

\section{EXPERIMENT 1}

\section{Method}

Stimulus preparation. Our previous studies of craniofacial growth validated a mathematical transformation which describes only one type of age-related change, namely the remodeling of the craniofacial complex throughout roughly the first 20 years of life. With respect to other age-related changes, such as wrinkling, a formal transformation is not presently known by which those changes can be generated. This fact posed a serious problem for the present investigation: At the outset, we had to find a procedure for systematically generating various stages of wrinkles.

A similar problem confronts an artist who is trying to portray a person at a particular age. To arrive at a successful portrait, the artist must depict those facial characteristics that have typically developed by that age. But how does the artist discover which facial properties are characteristic of a given age? One source of such information is provided by the work of Burne Hogarth (1965), an artist who has studiously observed the effects of growth and aging on the human head. In part of his book, Hogarth (1965, pp. 96-107) attempts to describe and portray typical changes in craniofacial appearance at different ages. His examination of growth and aging reflects both knowledge of craniofacial anatomy as well as careful observation of faces throughout the life span. Hogarth's illustrations, therefore, served as an informal model of various stages of wrinkles and other aging characteristics on which the construction of facial profiles for the present study was based. A secondary goal of the first experiment, however, was to validate Hogarth's drawings as perceptually adequate portrayals of the intended ages.

The facial profiles used in the present experiments were prepared in the following manner: Hogarth's profiles of a male at ages $15,30,50$, and 70 were traced by an experienced artist. 


\section{AGE PORTRAYED BY WRINKLES (YR)}

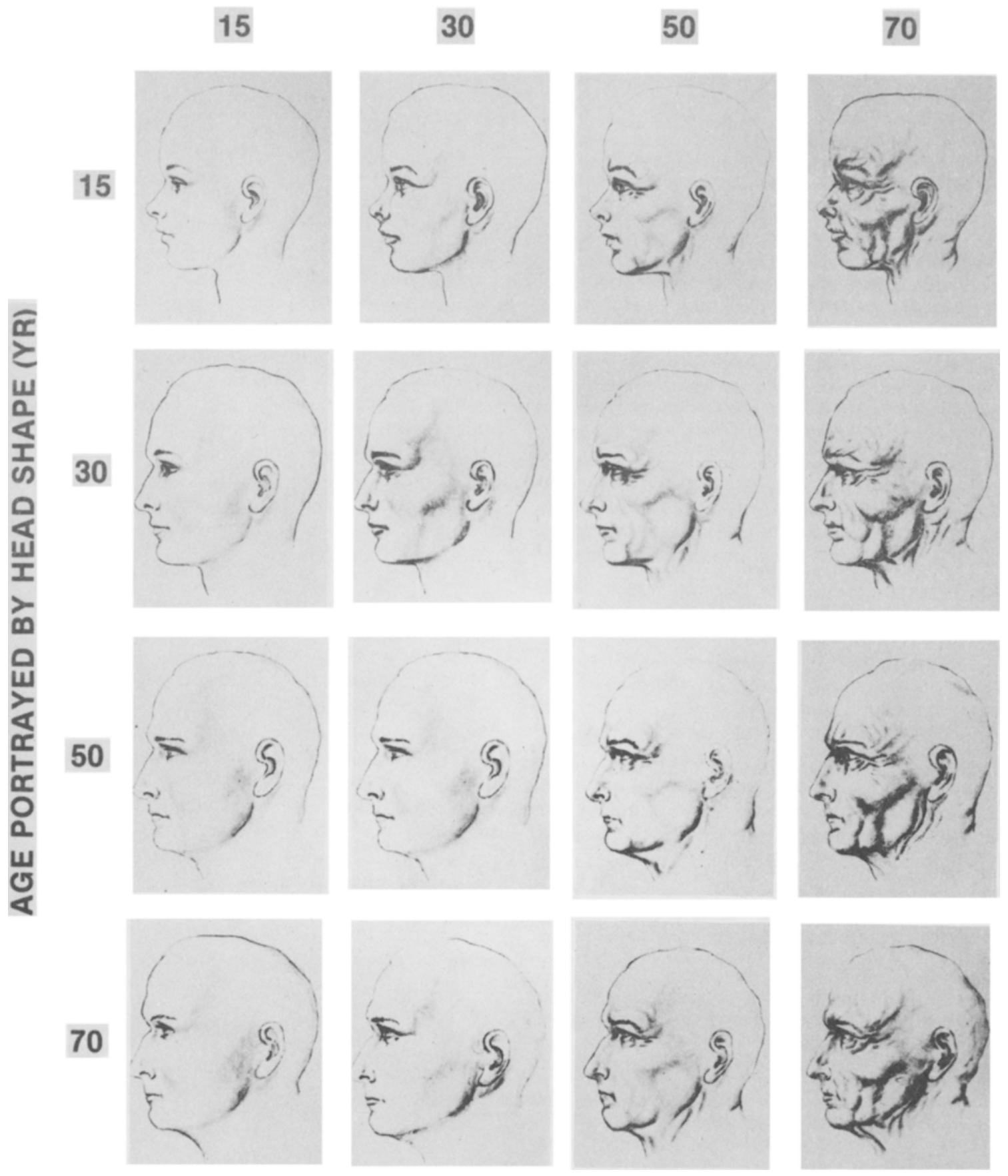

Figure 1. The 16 profiles systematically varying four levels of head shape and four levels of wrinkles, based on the work of the artist Burne Hogarth. 
Twelve additional profiles were then prepared by tracing the outline (head shape) of each of the four original Hogarth profiles and systematically substituting the internal detail (wrinkles) appropriate to profiles at other ages. ${ }^{3}$ Thus, each of the four head shapes was drawn with each of the four sets of wrinkles. Figure 1 shows the 16 resulting profiles. (In referring to particular profiles, HS stands for head shape and W stands for wrinkles.)

The 16 profiles were photographed individually and mounted onto slides which projected as black lines on a white background.

Subjects. Twenty-five undergraduates at the University of Arkansas, Little Rock, participated in this experiment for course credit.

Procedure. Slides of the profiles were displayed one at a time for approximately $8 \mathrm{sec}$. During that period, subjects recorded their estimates of each person's age, in years. The entire set of 16 profiles was presented to five groups of five observers. Different random orders were used with each group.

\section{Results and Discussion}

Experiment 1 examined the relative importance of changes in head shape and wrinkling on observers' judgments of the age level of a face. However, before considering the results with respect to the question of primary interest, it is necessary to determine that the four portraits traced from Hogarth's book (HS15-W15, HS30-W30, HS50-W50, and HS70-W70) were satisfactory representations of the ages intended by the artist. The mean age ratings computed over subjects and presentations are displayed in Table 1. Inspection of these means shows that Hogarth was quite successful in his attempts to produce information about the age level of faces: First, the marginal means for both dimensions, head shape and wrinkling, increase sharply in the direction intended by Hogarth. Second, each of the mean estimated ages of the profiles along the diagonal are close (within 5 years) to the intended ages.

In regard to the use of changes in head shape and wrinkling as information about age level, the mean age ratings in Table 1 show that both characteristics had a marked effect on subjects' age judgments. This observation was substantiated by a two-factor analysis of variance (Level of Head Shape by Level of Wrinkles); the main effects of head shape $[\mathrm{F}(3,72)=$ 134.28] and wrinkles $[F(3,72)=390.94]$ were significant beyond the .001 level. But the average range of head shape at each level of wrinkles was 22.6 years $\left(\overline{\mathrm{X}}_{\mathrm{HS70}}-\overline{\mathrm{X}}_{\mathrm{HS15}}\right)$. This was considerably smaller than the average range of wrinkles at each level of head shape, 42.9 years $\left(\bar{X}_{W 70}-\bar{X}_{W 15}\right)$. The seemingly greater impact of wrinkle information can also be seen in the mean age levels for the two profiles where head-shape and wrinkle ages are in greatest conflict, HS15-W70 and HS70-W15; for the former profile, the mean age estimate was nearly identical to the level of wrinkles, whereas the age estimates for the latter profile appeared to be a compromise between the levels of wrinkles and head shape.

These observations might be interpreted simply as suggesting that the changes across the four levels of wrinkles have a greater effect on age judgments than do changes in head shape. Such a conclusion, however, ignores the interaction between head shape and wrinkles $[F(9,216)=21.32 ; p<.001$ (cf. Table 1)]. This interaction can be explained in light of the relative rates of changes in head shape and wrinkling throughout the life span. Typically, at each level of head shape, the change in perceived age between wrinkle ages 15 and 30 years is small relative to the change between wrinkle ages 30 and 50 years or 50 and 70 years. This pattern of age judgments would be expected given the temporal development of wrinkling changes in facial appearance; only minor changes in the skin occur between the ages of 15 and 30 years compared to those occurring after 30 . All profiles with a level of wrinkles above 50 years, but one (HS15-W50), had a mean perceived age very near or above 50 years. Thus, the appearance of wrinkles after the age of 30 had a strong effect on the perceived age level of the faces.

An analogous interpretation of this interaction with respect to changes in head shape is also plausible. Most changes in head shape are completed prior to age 30. Although Hogarth's drawings of faces above the age of 30 depict some profile changes (e.g., due to edentation), their effect on perceived age should have been and was less significant than those occurring prior to age 30 , since below age 30 head shape is an important source of information about craniofacial age. On the other hand, changes in head shape had only a marginal effect on the perceived age of faces with severe wrinkling. This latter result is intuitively plausible as people are not likely to judge a highly

Table 1

Mean Absolute Age Judgments and Standard Deviations for Faces Depicting Four Levels of Wrinkles and Head Shape

\begin{tabular}{|c|c|c|c|c|c|c|c|c|c|c|}
\hline \multirow{3}{*}{$\begin{array}{c}\text { Age Level } \\
\text { Portrayed by } \\
\text { Head Shape }\end{array}$} & \multicolumn{10}{|c|}{ Age Level Portrayed by Wrinkles } \\
\hline & \multicolumn{2}{|c|}{15} & \multicolumn{2}{|c|}{30} & \multicolumn{2}{|c|}{50} & \multicolumn{2}{|c|}{70} & \multicolumn{2}{|c|}{ Mean } \\
\hline & $\mathbf{M}$ & SD & $\mathbf{M}$ & SD & $\mathbf{M}$ & SD & $\mathbf{M}$ & SD & $\mathbf{M}$ & SD \\
\hline $\begin{array}{c}15 \\
30 \\
50 \\
70 \\
\text { Mean }\end{array}$ & $\begin{array}{l}12.8 \\
23.2 \\
35.5 \\
40.0 \\
27.9\end{array}$ & $\begin{array}{l}4.30 \\
4.51 \\
6.62 \\
9.78 \\
6.30\end{array}$ & $\begin{array}{l}13.1 \\
26.1 \\
38.8 \\
45.4 \\
30.6\end{array}$ & $\begin{array}{l}4.20 \\
5.51 \\
9.23 \\
7.68 \\
6.66\end{array}$ & $\begin{array}{l}32.8 \\
49.2 \\
55.0 \\
57.0 \\
48.5\end{array}$ & $\begin{array}{r}18.90 \\
11.04 \\
7.60 \\
8.77 \\
11.58\end{array}$ & $\begin{array}{l}68.7 \\
70.3 \\
69.1 \\
75.3 \\
70.8\end{array}$ & $\begin{array}{r}15.57 \\
10.83 \\
11.09 \\
9.28 \\
11.69\end{array}$ & $\begin{array}{l}31.8 \\
42.2 \\
54.4 \\
54.4 \\
44.4\end{array}$ & $\begin{array}{r}10.74 \\
7.97 \\
8.63 \\
9.06 \\
9.06\end{array}$ \\
\hline
\end{tabular}


wrinkled face as young, regardless of its head shape.

Experiment 2 explored this interaction between head shape and wrinkles more directly by assessing observers' sensitivity to the coordination of these two general sources of age-related changes.

\section{EXPERIMENT 2}

Students of growth and aging have typically observed considerable coordination in changes over a number of local physical dimensions. In this sense, a "characteristic" relationship between head shape and degree of wrinkling can be said to exist at each general age level. Hogarth (1965) paid particular attention to these relations. There are, of course, individual variations in the coordination of sources of age-level characteristics: Some children have adultshaped heads and some elderly looking people have smooth, young-looking skin. As noted in the introduction, extreme discoordination among age-related characteristics is often a sign of abnormality, as in the case of progeria, which can result in wrinkling of a young child's skin.

While physical coordination of age-level indicants does occur normally, the perceptual salience of such relations is a different issue. The facial profiles used in the first experiment are appropriate stimuli with which to test observers' sensitivity to the relations between head shape and degree of wrinkling, since they include faces with levels of head shape and wrinkles that are coordinated with one another as well as faces with various amounts of discoordination between the two facial dimensions.

Ratings of perceived abnormality and conflict between the two dimensions were collected to assess observers' sensitivity to this coordination. If discoordination is quite salient and is seen as abnormal, we would expect low abnormality ratings on the faces in which the two dimensions depict the same age and increasingly higher abnormality ratings as the difference in ages depicted by the two dimensions increases. If, however, the discoordination is only moderately salient or is not seen as indicative of an abnormal condition, the above pattern of ratings might be obtained if observers were instructed to attend to head shape and skin wrinkling as indicators of age and to rate the conflict between them.

\section{Method}

Stimuli. The slides used in Experiment 1 were employed in both rating tasks.

Subjects. Forty undergraduate psychology students at the University of Arkansas, Little Rock, participated for course credit. None of these students was tested in the previous experiment.

Procedure. Experiment 2 is composed of a conflict rating task and an abnormality rating task. Twenty subjects, who were tested in four subgroups of five, participated in each rating task. Within each task, each subgroup viewed the 16 profiles in a different random order.
For the conflict rating task, the observers were instructed to rate the apparent degree of conflict between head shape and level of wrinkles in terms of the age level represented by each dimension. A rating scale ranging from 1 to 5 was used; a rating of 1 indicated that no conflict was observed, and a rating of 5 indicated that a large amount of conflict was seen.

For the abnormality rating task, observers were told that they were going to be shown profiles of people who had grown and aged normally and profiles of people who had undergone abnormal growth or aging. Their task was to rate the severity of any anomaly, using a 5-point rating scale; a rating of 1 indicated that no anomaly appeared to be present (i.e., normal growth and aging), and a rating of 5 indicated that a severe anomaly was present.

\section{Results and Discussion}

The second experiment examined whether observers were sensitive to the relations among various sources of age-level information. This was assessed using tasks in which subjects rated the apparent conflict between the age level indicated by the outline of the profile and the stage of wrinkling (conflict rating task) or whether the person appeared to have grown in a normal manner (abnormality rating task). The mean conflict ratings for the 16 profiles are presented in Table 2.

A two-factor analysis of variance showed that the main effects of head shape $[F(3,57)=11.25]$ and wrinkling $[\mathrm{F}(3,57)=7.32]$, and their interaction $[F(9,171)=21.22]$, were significant beyond the .001 level. With two exceptions (profiles HS30-W15 and HS15-W30), the mean conflict ratings were lowest for the profiles whose head-shape and wrinkle ages were identical. As the difference between the ages portrayed by wrinkling and head shape increased, the mean conflict judgments rose sharply: With 0 year's difference in the intended ages in the two variables, the mean conflict rating was 1.69; with 15 years' difference, 1.70 ; with 20 years' difference, 2.39 ; with 35 years' difference, 3.25 ; with 40 years' difference, 2.60; and with 55 years' difference, 3.48. That the means for 0 and 15 years' difference were nearly identical is likely due to the fact that the two profiles comprising the 15-year difference measure (HS30-W15, HS15-W30) showed little difference in the amount of wrinkling, since wrinkling does not normally begin

Table 2

Mean Conflict Ratings for Faces Depicting Four Levels of Wrinkles and Head Shape

\begin{tabular}{cccccc}
\hline $\begin{array}{c}\text { Age Level } \\
\text { Portrayed }\end{array}$ & \multicolumn{5}{c}{ Age Level Portrayed by Wrinkles } \\
\cline { 2 - 6 } $\begin{array}{c}\text { by Head } \\
\text { Shape }\end{array}$ & 15 & 30 & 50 & 70 & Mean \\
\hline 15 & 1.45 & 1.60 & 4.30 & 4.30 & 2.91 \\
30 & 1.80 & 1.85 & 2.25 & 2.94 & 2.21 \\
50 & 2.20 & 2.50 & 1.85 & 2.35 & 2.23 \\
70 & 2.65 & 2.25 & 2.45 & 1.60 & 2.23 \\
Mean & 2.03 & 2.05 & 2.71 & 2.80 & 2.40 \\
\hline
\end{tabular}

Note $-1=$ no conflict, $5=$ extreme conflict 
Table 3

Mean Abnormality Ratings for Faces Depicting Four Levels of Wrinkles and Head Shape

\begin{tabular}{cccccc}
\hline $\begin{array}{c}\text { Age Level } \\
\text { Portrayed } \\
\text { by Head }\end{array}$ & \multicolumn{5}{c}{ Age Level Portrayed by Wrinkles } \\
\cline { 2 - 6 } Shape & 15 & 30 & 50 & 70 & Mean \\
\hline 15 & 1.80 & 1.75 & 3.85 & 4.30 & 2.93 \\
30 & 1.05 & 1.50 & 2.30 & 3.45 & 2.08 \\
50 & 1.25 & 1.70 & 2.35 & 3.30 & 2.15 \\
70 & 2.05 & 2.20 & 2.00 & 2.90 & 2.29 \\
Mean & 1.54 & 1.79 & 2.63 & 3.49 & 2.36 \\
\hline
\end{tabular}

Note $-1=$ normal, $5=$ highly abnormal

prior to the age of 30 . As a result, both levels of wrinkles should be, and evidently are, perceived as appropriate for either head shape. However, the dramatic increase in the conflict ratings when the two age-level dimensions differ by more than 15 years indicates that, when prompted, subjects do notice the relationship between head shape and wrinkling.

The outcome of the abnormality rating task offered only weak support for this conclusion. An analysis of variance on the abnormality ratings showed that the effects of head shape $[F(3,57)=13.79]$ and wrinkling $[F(3,57)=51.38]$, and their interaction $[F(9,171)$ $=7.80]$, were significant beyond the .001 level. Table 3 presents the mean abnormality ratings.

The hypothesis that the abnormality ratings would be lowest for the profiles in which head-shape and wrinkle age coincided was not sustained. In general, the mean abnormality ratings increased with greater levels of wrinkles. Still, certain aspects of these data suggest that, with younger levels of head shape, alterations of the temporal coordination among facial dimensions are considered valid indications of a craniofacial anomaly. The younger shaped faces with heavy wrinkling are usually seen as quite abnormal, although the slightly wrinkled older shaped faces are seen as relatively normal. This outcome should have been expected: Heavy skin wrinkling is not always found among older people, even though wrinkling is usually thought of as a characteristic of old age (cf. Hogarth, 1965). Some people are able to stave off wrinkling as a result of cosmetic treatment, surgery (e.g., face-lifts), or climatic conditions. But this variability of wrinkling in no way diminishes the importance of wrinkles as a source of age-level information; when severe wrinkling is present, subjects usually judge the person to be quite old, regardless of head shape (Table 1). On the other hand, heavy wrinkling among children is, in fact, a symptom of a severe abnormality, progeria. We cannot, however, dismiss the possibility that subjects tended to judge wrinkles as an anomaly. Although wrinkles, wattles, bags, and creases are normal products of the aging process, it may be that our subjects, who had had no special training in the study of growth and aging, did not appreciate this fact. For this reason, the results of the abnormality task must be interpreted with caution. [The asymmetries in conflict ratings and abnormality ratings between profiles with young head shape and old wrinkles (HS15-W50, HS15-W70) and old head shape and young wrinkles (HS50-W15, HS70-W15) may well be a consequence of the fact that wrinkling is not always a product of the aging process.]

The pattern of conflict ratings shows that observers are sensitive to the relations among two sources of age information-head shape and wrinkling. But the conflict rating task does not address directly the issue of whether such relations constitute effective information for the perception of craniofacial age level. A reexamination of the data from the agerating task (Experiment 1), in conjunction with the results of the second experiment, has shed some light on this question.

The faces in which head-shape and wrinkle ages were discoordinated provided somewhat ambiguous information-ambiguous in the sense that the two dimensions suggest different ages. One possibility is that such ambiguity should manifest itself in a large amount of intersubject variability in perceived age level. Similarly, the faces that were seen as being rather abnormal might also be hard to judge for age and show high intersubject variability. To test this prediction, we examined the standard deviations of the age estimates for each of the profiles (Table 1) and the correlations among the mean age estimates, the standard deviations of age estimates, and the mean conflict and abnormality ratings on the 16 faces (Table 4).

Inspection of the standard deviations of the age estimate for the 16 profiles shows that the standard deviations tended to increase with increasing levels of head shape and wrinkling. This finding might be attributed to the apparently greater variation in the outcome of growth and aging processes as chronological age increases. Of greater interest is the comparison of the standard deviations of age estimates for the profiles with no conflict between head-shape

Table 4

Product-Moment Correlation Coefficients of Mean Age Judgments (Experiment 1), Standard Deviations of Age Judgments, Mean Conflict Ratings (Experiment 2), and Mean Abnormality Ratings (Experiment 2)

\begin{tabular}{|c|c|c|c|}
\hline & \multicolumn{2}{|c|}{ Age Estimates } & \multirow{2}{*}{$\begin{array}{c}\text { Mean } \\
\text { Conflict } \\
\text { Rating }\end{array}$} \\
\hline & Mean & SD & \\
\hline SD of Age Estimates & .506 & & \\
\hline Mean Conflict Rating & .343 & .931 & \\
\hline Mean Abnormality Rating & .657 & .853 & .784 \\
\hline
\end{tabular}

Note-For a one-tailed test, $N=16, .05$ level is $.426, .01$ level is .574, and .001 level is .623. 
and wrinkle age (representative profiles) and profiles with a difference in head-shape and wrinkle age (nonrepresentative profiles): The mean standard deviation for the representative profiles was 6.67 , while the mean for the 12 remaining nonrepresentative profiles was 9.85 . When the latter statistic was broken down by the amount of conflict (in years), the following mean standard deviations were obtained: 15 years' conflict, $4.36 ; 20$ years', 10.03; 35 years', 12.76; 40 years', 9.26; 55 years', 12.68. Beyond 15 years' conflict, the standard deviations rise markedly above those obtained for the representative profiles. (As in the conflict and abnormality tasks, we suspect that the profiles with 15 years' conflict were seen as fairly representative, since wrinkling generally has little effect on the face prior to age 30.) This pattern of results is at least consistent with our speculation that conflicting age information should increase the amount of variability in subjects' age estimates compared to the situation in which the different sources are in agreement. Moreover, the high correlations (Table 4) between the standard deviations of the age estimates and either the conflict ratings $(r=.931)$ or the abnormality ratings $(r=.853)$ offer additional support for the hypothesis that the relations between head-shape age and wrinkle age are apprehended by observers when they attempt to estimate a person's age from his or her facial appearance. ${ }^{4}$

\section{GENERAL DISCUSSION}

As a person grows and ages, many changes in facial appearance provide potentially useful information about the person's age. The present study examined two general types of changes: (1) the global remodeling of head shape that occurs throughout the first 20 years of life, and (2) the development of wrinkles (see Footnote 3), which becomes noticeable during adulthood. Experiment 1 demonstrated that observers are sensitive to both sources of craniofacial change in making age judgments. But more importantly, an interaction between levels of head shape and wrinkles revealed that the effect of either source of change on perceived age level depended upon the level of the other source of change. That observers appear sensitive to the coordination of the two dimensions of craniofacial change motivated the second experiment. The subjects were instructed to focus on head shape and wrinkles of each face and to indicate whether the two dimensions portrayed the same age or different ages (conflict rating task) or whether the face appeared to have a growth- or agingrelated anomaly (abnormality rating task). The outcome of the conflict rating task substantiated the results of Experiment 1: In general, the conflict ratings increased with the disparity between head-shape and wrinkle ages. A reexamination of the age ratings in Experiment 1 revealed a strong correlation between the standard deviations of the age estimate and the conflict rating for each profile, thereby offering support for the hypothesis that observers utilize various facial characteristics in making age judgments. The results of the abnormality task furnished only weak support for the hypothesis that observers perceive this "conflict" as indicative of a craniofacial anomaly.

The outcomes of both experiments point toward the same conclusion. In making judgments about the age of a person based on facial appearance, observers are sensitive to the overall profile contour (head shape) as well as facial wrinkling. However, the data also show that neither source of change is apprehended independently of the other; rather, relations among the various age-level dimensions constitute information for perceivers about the age level of the person and, perhaps, even the existence of agingrelated craniofacial anomalies. We regard this conclusion as but another example of the complex relations to which perceivers are sensitive (Gibson, 1966; Pittenger, Shaw, \& Mark, 1979; Shaw, Turvey, \& Mace, in press, Turvey \& Shaw, 1979). Thus, age level of faces is specified by an abstract relationship defined over various facial dimensions. The present study, though far from definitive, has shown that the relations between profile contour (head shape) and the appearance of the skin (wrinkling) constitute an important source of information about craniofacial age. It is quite likely that the relationships involved are far more complex than even those suggested by the present set of experiments. Still, this work emphasizes the global context in which each source of craniofacial change must be evaluated to delineate information specific to the age level of a given face.

\section{REFERENCES}

Brunswik, E. Perception and the representative design of psychological experiments. Berkeley: University of California Press, 1956.

EnLow, D. The human face: An account of the postnatal growth and development of the craniofacial skeleton. New York: Hoeber Medical Division, Harper \& Row, 1968.

Gibson, J. J. The senses considered as perceptual systems. Boston: Houghton Mifflin, 1966.

HogARTH, B. Drawing the human head. New York: WatsonGuptill, 1965.

KонN, R. R. Principles of mammalian aging. Englewood Cliffs, N.J: Prentice-Hall, 1971.

MARK, L. S. A transformational approach toward understanding the perception of growing faces. Unpublished doctoral dissertation, University of Connecticut, 1979.

Moore, W. J., \& LAvelle, C. L. B. Growth of the facial skeleton in the hominoidea. New York: Academic Press, 1974.

PICK, J. F. Surgery of repair: Principles, problems and practice. London: Lippincott, 1949.

Pittenger, J. B., \& Shaw, R. E. Aging faces as viscal-elastic events: Implications for a theory of nonrigid shape perception. 
Journal of Experimental Psychology: Human Perception and Performance, 1975, 1, 374-382.

PitTinger, J. B., Shaw, R. E., \& Mark, L. S. Perceptual information for the age-level of faces as a higher-order invariant of growth. Journal of Experimental Psychology: Human Perception and Performance, 1979, 5, 478-493.

Shaw, R. E., Turvey, M. T., \& Mace, W. Ecological psychology: The consequence of a commitment to realism. In W. Weimer \& D. Palermo (Eds.), Cognition and the symbolic processes II. Hillsdale, N.J: Erlbaum, in press.

Todd, J. T., Mark, L. S., Shaw, R. E., \& Pittenger, J. B. The perception of growth. Scientific American, in press.

Turvey, M. T., \& Shaw, R. E. The primacy of perceiving: An ecological reformulation of perception as a point of departure for understanding memory. In L.-G. Nilsson (Ed.), Perspectives on memory research: Essays in honor of Uppsala University's 500th anniversary. Hillsdale, N.J: Erlbaum, 1979.

VIIDIK, A. Functional properties of collagenous tissues. In D. A. Hall \& D. S. Jackson (Eds.), International review of connective tissue research (Vol. 6). New York: Academic Press, 1973.

\section{NOTES}

1. Although age judgments are measured using the same temporal units as chronological age, namely years, such age judgments are intended as a measure of what we call perceived age, which is a perceptual (as opposed to physiological) measure of developmental age.

2. Throughout the remainder of this paper, the terms "wrinkling" or "wrinkles" will refer to all of these changes.

3. It was determined that choosing a profile younger than 15 years created difficulties in superimposing the wrinkles of an old man on a facial outline of markedly different facial proportions. The decision not to use profiles younger than 15 years did minimize the amount of change in head shape. Still, it did not seem to affect the essential outcome of the experiment.

4. The results of these two experiments bear on an important methodological issue. Brunswik (1956) argued that psychology should use "representative" designs involving only naturally occurring stimuli, rather than systematic designs which include stimuli that are not representative of those actually occurring in life. The design used in the present study is obviously systematic: All levels of wrinkling and head shape were combined, independently of their representativeness.

The results illustrate the dangers of such designs. High intersubject variability in age judgments was found among the least representative faces. Therefore, it is not legitimate to interpret the statistically significant interaction of wrinkling and head shape as indicative of individual observers' weighting of cues for age level. While factorial designs are quite common in perception research, standard deviations are rarely reported.

However, one value of systematic designs was demonstrated. In some studies, the factorial combination of levels of several stimulus variables will produce displays which, while not representative of normal objects, do portray rare, but naturally occurring, meaningful objects. The faces with heavy wrinkling and a youthful shape are such a case. In such situations, it may be useful to examine whether or not observers are sensitive to the coordination of stimulus variables and correctly perceive the nature of the anomaly. Since no new stimuli are required to test these possibilities, such data can be readily collected.

(Received for publication July 10, 1979; revision accepted November 19, 1979.) 